\title{
Performance Analysis of Packet Switched All-Optical Networks
}

\author{
Marko Lackovic, Cristian Bungarzeanu \\ Telecommunication Laboratory (STI-ITOP-TCOM), Ecole Polytechnique Fèdèrale de Lausanne (EPFL) \\ CH-1015 Lausanne, Switzerland \\ Tel: $+4 / 2169356$ 76. Fax: +41 216932683 , e-mail:\{ marko.lackovic;cristian.bungarzeanu\}@epfl.ch
}

\begin{abstract}
All-optical packet switching has been intensively investigated in recent years as an alternative to static, crossconnect based networks. Several switch architectures have been proposed, all of them using buffers made of fibre delay lines. This paper addresses the basic concepts of the packet switching in the optical domain and describes an analytical approach to evaluate the end-to-end performance of networks employing slotted (fixed length) optical packets. Thus, for a given topology and traffic matrix, the end-to-end cell loss ratio is computed assuming an uncorrelated traffic. A network dimensioning procedure relying on this approach is also presented.
\end{abstract}

\section{INTRODUCTION}

The trend of unifications marked the last few years of transmission network evolution. Differences between transmission and commutation/switching in the network are not so visible any more. Future networks are often seen as intelligent content and service providers. The vision doesn't include any strict borders between the access and core network, and multiplexing hierarchies on the way, as in the case of the PDH and Sonet/SDH technologies employed in the voice communications. This evolution is the consequence of convergence of voice and data traffic. Voice is inherently based on the circuit switching, but data, mostly originating from the Internet, is packet based. Transmission network was up-to-now based on the circuit switching of WDM channels with the configurable switches as the (prospective) way to introduce time dimension and dynamics into traditionally static transmission network.

The idea of packets for the electronic layer slowly started to migrate into the transmission network, thus eliminating the need for a circuit-switching oriented framing protocol like Sonet/ SDH. The reason for packet switching paradigm [1] introduction to the transmission network is better resource utilization, due to statistical multiplexing which was proven as superior to traditional static multiplexing. This is of great importance for dealing with the self-similar features which can be found in aggregated traffic [2]. Moreover, packet based switching offers greater granularity than the rarely reconfigurable channels in the OCS. Greater granularity means more flexibility in the reconfiguration and service provision, which is very important in the multi-service network paradigm, and assuring quality of service on the physical level.

\section{TECHNOLOGY ISSUES}

The technology is the major obstacle for the optical packet switching (OPS) deployment. Gigabit rates are too high for the slowly improving speed of electronic circuits. Optical technology is thus a promising solution, but still in early phases of development.

The key technology issues that have to be addressed include fast optical packet header processing, switching and buffering. The semiconductor optical amplifiers (SOAs) are the key component which determines the cost effectiveness of the OPS as the competing technology to OCS.

OPS deployment depends on the switching matrix ability to support the required ns switching times. The criteria for selecting the suitable switching scheme for OPS includes following issues:

- Switching time - switching time for the OPS should be less than 100ns in order to keep short overhead,

- Throughput - should be large enough to support core network traffic,

- Signal degradation - includes optical loss and cross-talk. Important for scalability issues in the case where optical regeneration is not applied.

Switching schemes include space switches, broadcast-and-select-switches and wavelength routers.

Buffering issues are based on the problem of light trapping. This is achieved by introducing delay in fiber delay lines (FDLs), but the problems arise due to available discrete buffering times and very long fibers needed for longer buffering times (larger memories).

Other solutions between circuit and packet switching, like optical burst switching, evolved as the answer to technology issues. They could be deployed in the next few years, but the OPS remains the long term solution for expected higher traffic volumes and traffic unpredictability.

\section{PACKET HANDLING SCHEMES}

There are two different approaches to the OPS network design concerning the employed time frame. The first one called the synchronous scheme assumes that a packet can arrive to the switching fabric only in certain time

This work has been performed within the COST Action 266 "Advanced infrastructure for photonic networks", with the support of Swiss Federal Office for Education and Science. 
points. The time scale is divided into time slots. The other one called the asynchronous assumes that a packet can arrive to a switching fabric in any time point. Each scheme implies only the time frame, but not the size of the packet, and thus each can be combined with the fixed-length packets (cells), or variable length packets (Figure 1). However, the majority of proposed OPS networks are based either on the synchronous (slotted) network with cells, or asynchronous network with variable lengths packets.

unslotted, variable length unslotted, variable length
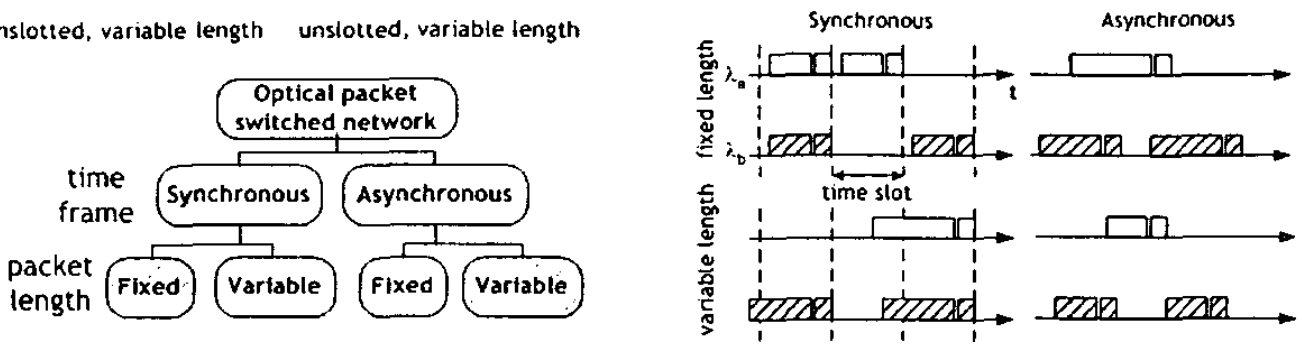

Figure 1. Mllustration of the packet handling schemes.

The synchronous network approach assumes synchronizer which aligns packets to time slot beginnings. A global synchronization has to be achieved, which represents a problem in large area networks (e.g. Pan- European network). Fixed length packets require fragmentation of large client packets what implies more overhead and makes the node architecture more complex due to reassembly. Bandwidth capacity waste is additionally achieved by transmitting payloads smaller than the header. On the other hand, this approach minimises blocking and simplifies the FDL based buffers and makes switch matrix reconfiguration. The order of the packet is easy to maintain. This solution is thus more feasible in the middle term perspective.

Asynchronous networks with variable packet length don't require fragmentation, nor aligning, but the blocking probability increases [3]. The packet header has to contain the packet length field.

The best packet handling scheme is difficult to determine. However, the variable length packets are considered to be a better solution, due to the inherent similarity with the IP packet as the clients for the optical layer.

\section{OPTICAL PACKET AND OPTICAL PACKET PROCESSING}

Optical packet generally has a payload part containing the client data, and the header. The header and payload are separated (time or wavelength domain), and thus bit rate independent. The header is processed in the electronic domain (due to the immaturity of the optical processing devices), but the payload is transmitted entirely in the optical domain. The header could be transported in a separate channel [4], but this solution can impose synchronization difficulties between headers and payloads.

Figure 2 depicts a general optical packet format.

\begin{tabular}{|c|c|c|c|c|}
\hline Synchronisation & Label & Guardtime & Payload & Guardtime \\
\hline
\end{tabular}

Figure 2. General optical packet format.

The following parts of the packet format are common to the majority of propositions:

- Synchronization - denotes the beginning of the packet. It is essential in asynchronous networks, but also needed in synchronous networks to resynchronize clocks and eliminate time jitter, what is needed to read the label.

- Label - contains destination address, length, priority, hop count, etc. The label should provide enough information to route the packet through the network, insure classes of service, and avoid loops. There have been several proposals to label encoding. Sub-Carrier Modulation (CORD [5], OPERA [6]) is based on the idea of amplitude modulated sub-carrier on the frequency higher than the payload frequency. The label is modulated into the sub-carrier (e.g. AM), but on the lower bit rate more suitable for electronic processing. The optical carrier is then modulated by the sub-carrier. The advantage of this approach is simultaneous transmission of the payload and the label.

- Guard time - time gap that eliminates overlapping of adjacent packets, which could be caused by unequal switching times, or time shifts in header processing (reading/writing).

- Payload - carries client data. The payload should be transparent to the optical channel speed, as well as to the format of the client data. IP datagrams are considered in most cases as the client data, but some other format could be carried by this field as well.

\section{CONTENTION RESOLUTION}

The efficiency of contention resolution mechanism has a major influence on the network efficiency in terms of packet loss ratio. This problem is solved on the IP layer (electronic domain) by using electronic buffers (RAM). 
This solution is unfeasible in the optical domain, and packer storage is achieved by delaying the signal in FDLs. OPS contention resolution has various solutions [1], but three can be identified as the basic ones (Figure 3):

1. Improve the capacity sharing by wavelength conversion (WASPNET),

2. Buffering based on FDLs (KEOPS),

3. Deflection routing to avoid or reduce buffering.
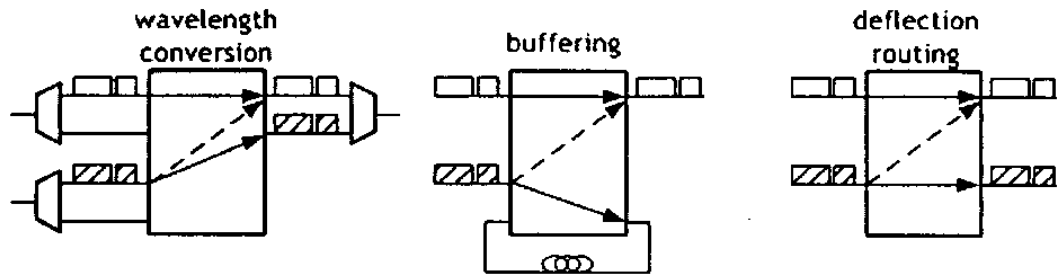

Figure 3. Contention resolution approaches in OPS.

\section{OCS vs OPS NETWORK DESIGN}

Network design assumptions include positions of network nodes, and traffic matrix. In both cases demands have to be routed to shortest physical paths. After that for the OCS case the capacity minimization should take place, and the logical topology should be created. This determines which routers will communicate directly over the physical layer, and which will communicate via some other router. This will cause the rearrangement of demands from the shortest paths. The protection and restoration schemes have to be taken into account in this phase. The channels have to be properly dimensioned to support the traffic demands and possible traffic fluctuations. After the demand positioning and channel dimensioning the wavelengths have to be assigned to communication channels. This is trivial in the case of full wavelength conversion, but gets complicated in the case of no wavelength conversion. The idea is to get a trade-off between the number of used wavelength converters and equipment usage. This is the end of the design phase and gives enough information for the physical topology construction (laying fiber in the cables). Node structure depends on the wavelength conversion and space switching possibilities.

In the OPS case the design phase is simpler, because the aggregation (achieved in the logical OCS topology design phase) is achieved in the OPS inherently by the packet switching mechanism. The problem is to determine the link capacities between OPS routers, which are able to support the traffic matrix, traffic flow characteristics (burstiness, self-similarity) and the additional flows in the case of deflection routing.

\section{OPS NETWORK STRUCTURE AND PERFORMANCES}

Assumptions included all-optical network with the optical packet switching capability in each node and the fixed length input packets (further referred as cells). Figure 4 shows the general network model. Each node has its own local data source/destination. Traffic demands between two nodes are supported by the physical WDM links.

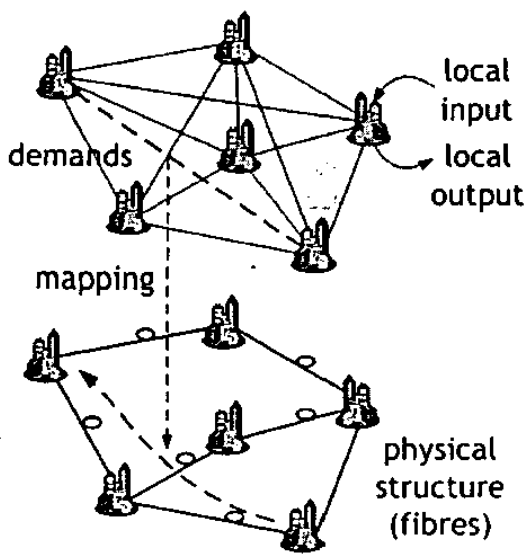

Figure 4. General network model.

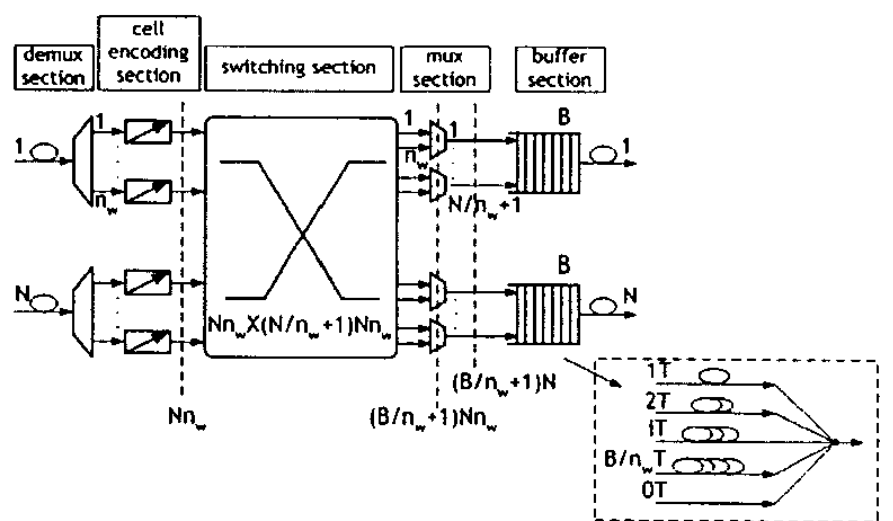

Figure 5. The switch matrix architecture with internal buffer structure. 


\subsection{Switch Architecture}

The model for the switch architecture was taken from [7]. Each switch has $N$ inputs and outputs, and comprises 5 parts - the demux/mux sections, cell encoding and the buffer section (Figure 5). The switch could be classified as the output buffered switch with wavelength conversions.

The demux section contains $N$ demultiplexers with $n_{w}$ exits, assuming that each input carries a WDM signal with $n_{w}$ wavelengths. The cell encoding section contains tunable wavelength converters, which choose the right wavelength according to the free space in buffers as described below. The use of wavelength converters is essential to minimize the number of necessary delay lines [7].

The core part is the $N \cdot n_{w} \times\left(B / n_{w}+1\right) \cdot N$ unblocking space cross-connect. The number of demultiplexers and wavelengths determine the number of switch inputs, while the buffer capacity determines the number of switch outputs.

Each buffer has the same capacity denoted as $B$, implying that $B$ cells can be stored in the buffer at the same time. A buffer is based on the fiber delay lines. The number of delay lines depends on the number of wavelengths used, and can be expressed as $B / n_{w}+1$ (Figure 5).

Delay introduced by the lines varies from 0 (direct connection to the output) to $B / n_{w} T, T$ being the time slot duration (slotted network assumption). Each delay line carries a multiplexed signal, and is able to store up to $n_{w}$ cells. It gives the total number of $B$ cells that can be stored. $n_{w}$ cells can exit the switch using the same exit during each time slot, what corresponds to the number of cells that can enter the switch during one time slot using the same input. There are $N$ buffers, one for each output implying that the number of switch outputs has to be $N n_{w}\left(B / n_{w}+1\right)$. Number of multiplexers is equal to the number of delay lines (including the zero time connection) or $N\left(B / n_{w}+1\right)$.

\subsection{Computer Model \& Calculations}

Input data is taken from the COST266 project. Five reference topologies have been analysed, along with a traffic model for calculating traffic matrix for each of the topologies. The proposition includes: basic topology (BT), core topology (CT), large topology (LT), ring topology (RT) and triangular topology (TT) [8].

Total traffic comprises three traffic classes - voice traffic, transaction data traffic and Internet traffic [9]. The actual traffic between nodes (cities) is calculated as a sum of these three traffic classes between them.

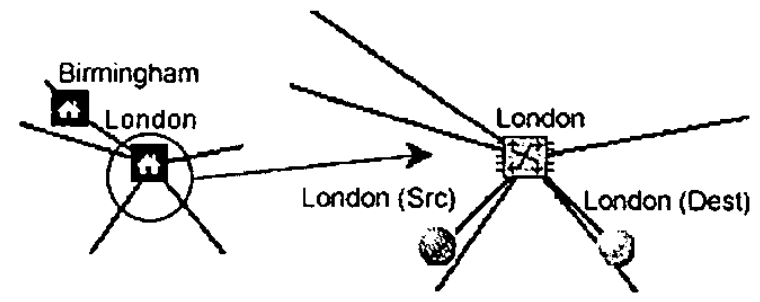

Figure 6 Node structure.

Network nodes correspond to cities involved in the topology. Each node contains an optical packet switch, optical packet source node, and optical packet destination node. Each node can be a traffic source and destination. Chosen topology determines physical connections between switches. Top-level nodes thus just serve as a wrapping for packet switching functionality based on the switch and source/destination nodes (Figure 6). Traffic matrix obtained from the described network model determines capacities of demands. Each demand is formed between a source and destination. Sources and destinations are connected to the switch by $10 \mathrm{~km}$ long links (arbitrary assumption).

Cell loss ration (CLR) is defined as the ratio of lost cells and all cells. The term lost cells includes all cells that were discarded because of the insufficient buffer capacities.

The calculations rely on the probabilities of founding a number of cells in some buffer in a point of time. The easiest way is to represent the number of cells in a buffer using a Markov chain, and to define transition probabilities. The analytic calculation of CLR and buffering times [10] has been implemented using the CANPC tool [11]. Buffering time calculation is based on delays introduced by queuing in output buffers in switches.

\section{PLANNING PROCEDURE}

A channel capacity of $40 \mathrm{Gbps}$ was assumed to reduce the complexity of the analytic algorithm, which depends on the number of used wavelengths. Number of required iterations of algorithm to cover all possible cases is equal to $(1+n)^{N}$ where $n$ stands for number of used wavelengths and $N$ denotes number of switch inputs. In order to keep the wavelengths low we chose this channel capacity. Number of wavelength used to calculate buffering time and CLR was 4 and 8 per fiber.

The planning procedure itself comprises two sets: 
1. Worst-case planning, and

2. Planning using the upper CLR constraint.

The term worst-case planning suggests that no considerations about the CLR have been made as network links have been dimensioned just to support the traffic demands. This implies that the number of fibers in each link depends just on the total capacity of demands using the link. The aggregate traffic capacities required on each link has been obtained by routing the traffic demands on the shortest paths.

The previous results for the worst-case network modeling can be improved in terms of reducing the demand CLR values under some upper CLR constraint. Figure 7 depicts the steps of the modeling procedure. Previous network dimensioning enters as initial solution to be improved.

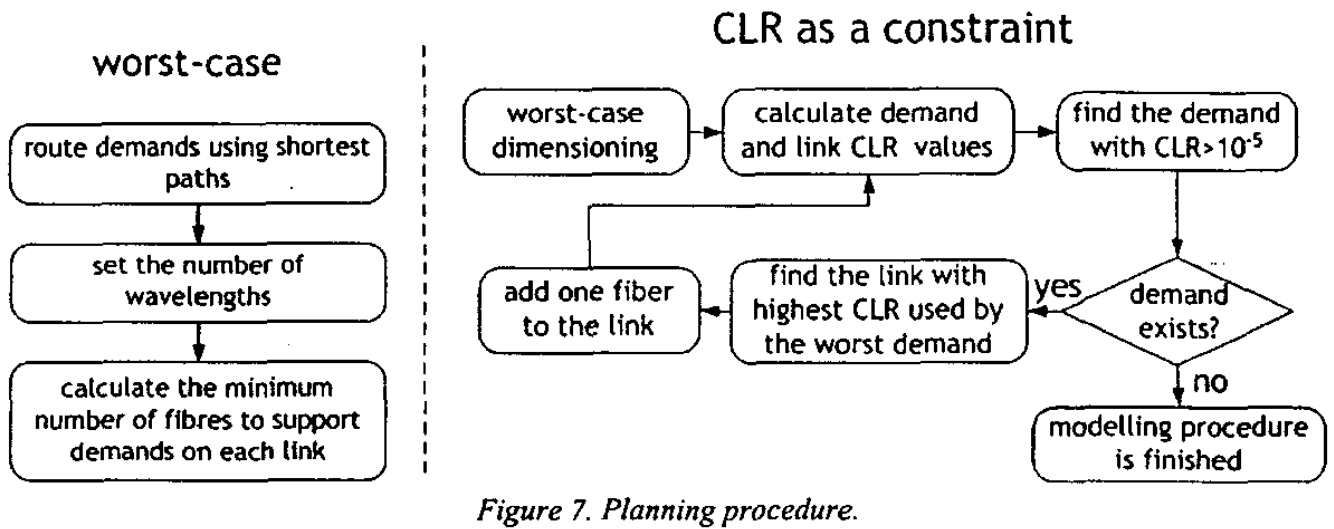

\section{RESULTS}

The CLR and buffering time calculations have been made for all topologies. Calculations have been made for 4 and 8 wavelength per fiber. The analysis of CLR and buffering time has been made on demand and on fiber basis. Results include mean and maximum values for CLR and buffering time for both categories. Buffering times are calculated relative to the time slot duration. Number of delay lines is set to 3 . The (S) label denotes optical packet source (link between the source and optical packet switch), while the (D) label denotes optical packet destination.

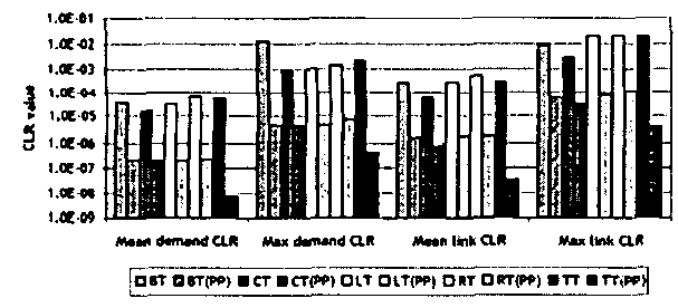

Figure 8. CLR values comparison (4 wavelengths).

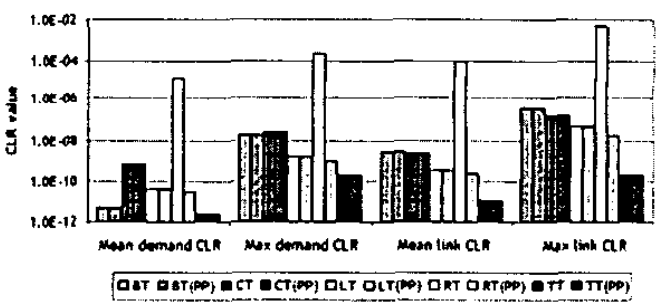

Figure 9. CLR values comparison (8 wavelengths).

Following graphs depict the time calculation group. RT one again shows the worst results caused by high link utilization, which was the cause of the high CLR. High utilization caused by high traffic aggregation introduces long waiting times in output buffers resulting high mean packet delay times. The TT has the lowest mean delay times due to low link utilization and low traffic aggregation.

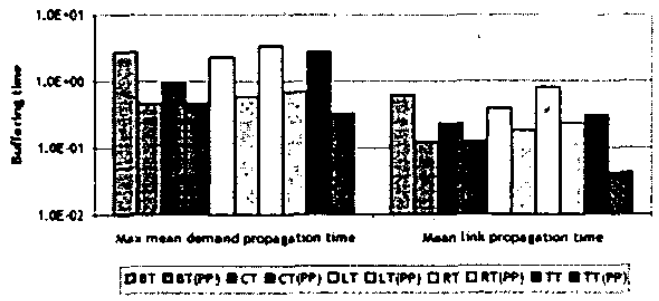

Figure 10. Time values comparison (4 wavelengths).

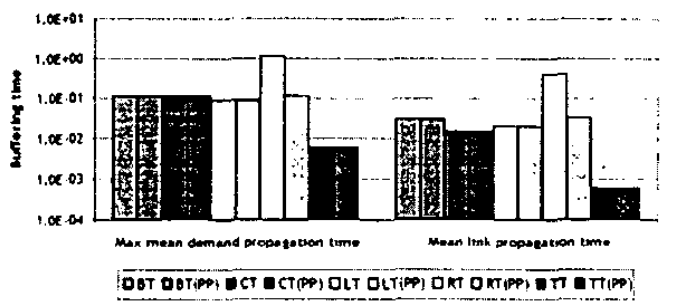

Figure II. Time values comparison ( 8 wavelengths).

\section{CONCLUSIONS AND FUTURE WORK}

This article gives a brief overview of the most important optical packet switching issues and proposes a planning 
procedure for OPS based network dimensioning. Cell loss ratio and buffering time analysis were the criteria for OPS network performance evaluation. Calculations are based on analytical procedures implemented in the CANPC tool and applied to Cost266 topologies. Final results include topology comparison and overview of improvements gained by the planning procedure.

\section{REFERENCES}

[1] S. Yao, B. Mukherjee, S. Dixit, "Advances in Photonic Packet-Switching: An Overview", IEEE Comm. Mag., February 2000, pp. 84-93.

[2] V. Paxson and S. Floyd, "Wide-area Traffic: The Failure of Poisson Modelling", IEEE/ACM Transactions on Networking, pp.226-244, June 1995.

[3] P.B.Hansen, S.L. Danielsen, K.E. Stubkjaer, "Optical packet switching without packet alignment", Proc. ECOC'98, pp. 591-592. Madrid, Spain, 1998.

[4] M. C. Chia et al. "Packet Loss and Delay Performance of Feedback and Feed-Forward ArrayedWaveguide Gratings-Based Optical Packet Switches With WDM Inputs-Outputs". IEEE JLT 19(9), September 2001.

[5] D. Chiraroni et al: Experimental validation of an all-optical network based on $160 \mathrm{Gbit} / \mathrm{s}$ throughput packet switching nodes, in ECOC 1998, pp. 573-574.

[6] D. Sahri et al: A Highly integrated 32-SOA gates optoelectronic module suitable for IP multi-terabit optical packet routers, in Proc. OFC '01, PD32.

[7] Danielsen, S.L., et al., "WDM Packet Switch Architectures and Analyisi of the Influence of Tuneable Wavelength Converters on Performance", Journal of Lightwave Technology, 15(2), February 1997.

[8] Lackovic, M., C. Bungarzeanu, An Approach to Planning and Performance Evaluation of Networks Based on Optical Packet Switching, Proc. of ONDM 2003, The 6th IFIP Working Conference on Optical Network Design \& Modelling, February 3-5, 2003, Budapest, Hungary, pp. 785-799.

[9] De Maesschalck, et al., "Pan-European Optical Transport Networks: an Availability-based Comparison (result of IST LION and COST 266)", submitted to the Photonic Network Communications.

[10] Lacković, M., C. Bungarzeanu, Planning Procedure and Performance Analysis of Packet Switched Alloptical Network, Proc. of ONDM 2003, The 6th IFIP Working Conference on Optical Network Design \& Modelling, February 3-5, 2003, Budapest, Hungary, pp. 253-271.

[11] Strahm, P. and C. Bungarzeanu, "Planing Tool for WDM Networks with All-Optical Packet Switching", Proceedings of WAON, June 13-14, 2001, Zagreb, Croatia, pp. 51-54. 